Comment. Math. Helv. 74 (1999) 530-547

(C) 1999 Birkhäuser Verlag, Basel

0010-2571/99/040530-18 \$1.50+0.20/0

Commentarii Mathematici Helvetici

\title{
Boundary slopes of knots
}

Marc Culler and Peter B. Shalen

Mathematics Subject Classification (1991). Primary 57M40, 57M25; Secondary 57M50, $57 \mathrm{~N} 10$.

\begin{abstract}
The results in this paper show that simple connectivity of a 3-manifold is reflected in the behavior of essential surfaces in exteriors of knots in the manifold. A corollary of the main theorem is that any non-trivial knot, with irreducible complement, in a homotopy 3-sphere must have two boundary slopes that differ by at least 2 . This statement is false for knots in a homology 3 -sphere. The main theorem itself applies more generally to knots in closed orientable 3 -manifolds with cyclic fundamental group.
\end{abstract}

\section{Introduction}

If $K$ is a knot in the 3-sphere, any minimal-genus spanning surface may be isotoped so as to meet the exterior $M=M(K)$ in a non-separating bounded essential surface. (See Section 1 for the definition of essential surface and of other terms used here.) Essential surfaces are useful in the study of the topology of the knot exterior $M$; for example, the existence of a non-separating bounded essential surface implies, via Papakyriakopoulos's work, that $\pi_{1}(M)$ is an HNN extension with a free associated subgroup. It was shown in [4] that if $K$ is non-trivial then $M$ always contains a connected bounded separating essential surface $F$. This implies the result, first conjectured by Neuwirth, that $\pi_{1}(M)$ is a non-trivial free product with amalgamation, and that the amalgamated subgroup is free. That $F$ separates $M$ is a consequence of the fact that its boundary slope, an element of $\mathbb{Q} \cup\{\infty\}$ that encodes the common isotopy class of all the components of $\partial F$, is non-zero.

It follows from a theorem of Hatcher's [6] that the set $\mathcal{B} K \subset \mathbb{Q} \cup\{\infty\}$ of all boundary slopes of essential surfaces is finite for any knot exterior $M$. The arguments of [4] show that this set has at least two elements if $K$ is non-trivial. The set $\mathcal{B} K$ has been computed for some families of knots. The first such computations were made for 2-bridge knots by Hatcher and Thurston [8]. Their methods were generalized by Hatcher and Oertel [7], who gave a procedure for computing $\mathcal{B} K$

Both authors are partially supported by a grant from the National Science Foundation. 
in the case that $K$ is a Montesinos knot.

The results in [8] led Hatcher and Thurston to ask whether boundary slopes are always even integers, and whether there always exist two boundary slopes that differ by more than 2. The first question was answered negatively in [1]. (More generally, Hatcher and Oertel showed in [7] that every rational number arises as a boundary slope for some Montesinos knot.) In the present paper we address Hatcher and Thurston's second question. We show that for a non-trivial knot $K$ in $S^{3}$, the diameter of $\mathcal{B} K$ is at least 2, i.e. that if $\infty \notin \mathcal{B} K$ then there are two elements $r, s \in \mathcal{B} K \subset \mathbb{Q}$ such that $r-s \geq 2$. This is included in Corollary 1.6 below, and may be regarded as a refinement of the main result of [4], insofar as the latter result bears on knots in $S^{3}$.

There is an important difference between the nature of the results about $\mathcal{B} K$ proved in this paper and those that were proved in [6] and [4]. The set $\mathcal{B} K$ is well-defined for any knot $K$ in an integer homology sphere. For a knot in an arbitrary closed orientable 3-manifold it is defined modulo integer translations and reflection about 0 . (In this paper, just as a matter of convenience, we define $\mathcal{B} K$ only for knots with irreducible complement.) Now, the results of [6] establish the finiteness of $\mathcal{B} K$ not only for a knot $K$ in $S^{3}$ but for a knot in an arbitrary closed orientable 3-manifold. Likewise, the arguments of [4] can easily be adapted to give a proof that if $K$ is a non-trivial knot in an arbitrary closed 3-manifold $\Sigma$, and if $M(K)$ does not contain an essential separating annulus or a non-separating torus, then $K$ has at least two boundary slopes. (This result is made explicit in [12].) By contrast, Corollary 1.7 below, which asserts that $\mathcal{B} K$ has diameter at least 2, requires the hypothesis that $\Sigma$ be a homotopy 3 -sphere. In fact, the discussion given below in 1.8 shows that for any $\epsilon>0$, there exist a homology 3-sphere $\Sigma$ and a non-trivial knot $K \subset \Sigma$ such that the diameter of $\mathcal{B} K$ is $<\epsilon$. Thus the results of this paper show that simple connectivity of a 3-manifold is reflected in the behavior of essential surfaces in exteriors of knots in the manifold. Our results are therefore relevant to the Poincaré Conjecture. We discuss this further, in a more general context, in Section 1.9.

Corollary 1.7 is derived from our main result, Theorem 1.1, which applies more generally to a closed, orientable 3 -manifold $\Sigma$ with a cyclic fundamental group. If $K$ is a non-cabled knot in $\Sigma$ such that $\infty \notin \mathcal{B} K$, Theorem 1.1 implies that either $\mathcal{B} K$ has diameter at least 2 or else $\Sigma$ has a genus-1 Heegaard splitting in which $K$ is a core curve for one of the Heegaard solid tori. (The actual statement of Theorem 1.1 is slightly stronger. For precise definitions of the terms used here, see Section 1.)

Theorem 1.1 is sharp: Nathan Dunfield has discovered that there is a hyperbolic knot $K$ in a 3 -manifold with cyclic fundamental group such that $\mathcal{B} K$ has diameter exactly 2. Dunfield's example is described in Example 1.4. In [5] Dunfield gives conditions under which the inequalities of Theorem 1.1 and Corollary 1.7 are strict.

The proof of our main result combines the techniques of [3] and [4] with properties of the norm on $H_{1}(\partial M ; \mathbb{R})$ which was introduced in Chapter I of [1]. Given 
the techniques of these earlier papers, the proof of the main theorem is relatively simple in the case of a hyperbolic knot. One of the refinements which are needed to handle the non-hyperbolic case is a generalization of the theory of Chapter I of [1] to manifolds with more than one boundary torus. This is developed in Section 2. Our results are stated in Section 1 and proved in Section 4, and a key topological lemma is proved in Section 3.

We thank Nathan Dunfield and Cameron Gordon for many helpful discussions. We are especially grateful to Dunfield for permission to present Example 1.4 in this paper.

\section{Notation and Main Results}

Suppose that $\Sigma$ is a closed connected orientable 3-manifold. A knot in $\Sigma$ will always be understood to be tame. If $K$ is any knot, a regular neighborhood $U$ of $K$ is a solid torus, the knot exterior $M=\overline{\Sigma-U}$ is a compact 3-manifold with boundary, and $\partial M=\partial U$ is a torus. We will say that $K$ is round if the manifold $M$ has a solid torus connected summand. In other words, $K$ is round if and only if $M$ is boundary-reducible.

In the case where the exterior $M$ of $K$ is irreducible, $K$ is round if and only if $M$ is a solid torus. Note that this implies that $\Sigma$ has a genus-one Heegaard splitting and is therefore homeomorphic to a (possibly trivial) lens space or to $S^{2} \times S^{1}$.

For any knot $K \subset \Sigma$, the regular neighborhood of $K$ and the exterior of $K$ are well defined up to ambient isotopy. In certain situations this will allow us to write $U(K)$ for a regular neighborhood and $M(K)$ for a knot exterior without risk of ambiguity.

If $U$ is a solid torus in $\Sigma$ and $M=\overline{\Sigma-U}$, we define a meridian of $U$ to be an oriented simple closed curve in $\partial M=\partial U$ which bounds an essential disk in $U$. We think of such a curve as being defined up to oriented isotopy. From this point of view, a knot has two meridians, which differ only in orientation. Furthermore, we shall not always distinguish between an isotopy class of oriented simple closed curves in $\partial M$ and the corresponding primitive class in $H_{1}(\partial M ; \mathbb{Z})$; thus a meridian may be regarded as an element of $H_{1}(\partial M ; \mathbb{Z})$. We define a framing for the solid torus $U$ in $\Sigma$ to be a basis $(\mu, \nu)$ of $H_{1}(\partial M ; \mathbb{Z})$, where $\mu$ is a meridian.

If $K$ is a knot in $\Sigma$, we shall often refer to a meridian of $U(K)$ or a framing for $U(K)$ as a meridian of $K$ or a framing of $K$, provided that no ambiguity arises.

In the case where $\Sigma$ is an integer homology 3-sphere, orientations of $\Sigma$ and $K$ give rise to a canonical framing $(\mu, \lambda)$ for $K$ in the following way. The orientation of $\Sigma$ restricts to an orientation of $M=M(K)$ which induces an orientation of $\partial M$. The kernel of the inclusion homomorphism $H_{1}(\partial M ; \mathbb{Z}) \rightarrow H_{1}(M ; \mathbb{Z})$ is a direct summand of $H_{1}(\partial M ; \mathbb{Z})=H_{1}(\partial U(K) ; \mathbb{Z})$, and is generated by an element $\lambda$ whose image in $H_{1}(U(K) ; \mathbb{Z})$ is the element defined by the given orientation of 
$K$. We may think of $\lambda$, which is called a longitude, as an oriented simple closed curve in $\partial M$ defined up to isotopy. For either choice of the meridian $\mu$ we have an ordered basis $(\mu, \lambda)$ for $H_{1}(\partial M ; \mathbb{Z})$; furthermore, there is a unique choice of $\mu$ for which this ordered basis determines the induced orientation of $\partial M$.

If $U$ is a solid torus in a general closed, orientable 3-manifold $\Sigma$, and if $M=$ $\overline{\Sigma-U}$, it follows from Poincaré duality that $\operatorname{ker}\left(H_{1}(\partial M ; \mathbb{Z}) \rightarrow H_{1}(M ; \mathbb{Z})\right)$ is infinite cyclic, and thus is contained in a unique direct summand $Z$. A generator of $Z$ will be called an external longitude for $U$. In the case that $\Sigma$ is an integral homology sphere the longitude of $U$ is an external longitude. In general, however, the kernel may be a proper subgroup of $Z$, and $Z$ may fail to be complementary to the summand generated by the meridian. For example, if $H_{1}(\Sigma)$ is infinite cyclic, and if a core curve of $U$ represents $k$ times a generator of $H_{1}(\Sigma ; \mathbb{Z})$, then $\operatorname{ker}\left(H_{1}(\partial M ; \mathbb{Z}) \rightarrow H_{1}(M ; \mathbb{Z})\right)$ has index $k$ in $Z$ and the meridian is a generator of $Z$, i.e. the meridian is an external longitude.

Suppose that $K$ is a knot in a closed orientable 3-manifold $\Sigma$, and let us set $M=M(K)$. If we fix a framing $(\mu, \nu)$ for $K$ then any oriented, homotopically nontrivial simple closed curve $\gamma$ in $\partial M$ represents a class $p \mu+q \nu$, where $p$ and $q$ are relatively prime integers. The element $p / q \in \mathbb{Q} \cup\{\infty\}$, called the slope of $\gamma$, depends only on the isotopy class of the simple closed curve $\gamma$, not on its orientation. This defines a bijection between isotopy classes of unoriented, homotopically non-trivial simple closed curves in $\partial M$ and elements of $\mathbb{Q} \cup\{\infty\}$. This bijection depends on the choice of a framing. Changing the framing has the effect of composing the bijection with an element of the dihedral group $D_{\infty}$ generated by $z \mapsto z+1$ and $z \mapsto-z$. Note that for the case of an oriented knot in an oriented integer homology sphere, we have a standard choice for this bijection arising from the standard framing.

Let $M$ be an orientable compact irreducible 3-manifold whose boundary components are tori. By an essential surface in $M$ we mean a tame, orientable, properly embedded 2-manifold no component of which is a 2-sphere or a surface which is parallel to a subsurface of $\partial M$. A connected essential surface $F$ is called a fiber if it is a fiber in some fibration of $M$ over $S^{1}$; it is called a semifiber if $M$ is a union of two twisted $I$-bundles $E_{1}$ and $E_{2}$ over non-orientable surfaces, such that $E_{1} \cap E_{2}=F$ and $F$ is the common associated $\partial I$-bundle of $E_{1}$ and $E_{2}$. We will say that an essential surface $F$ is strict if no component of $F$ is a fiber or a semifiber.

If the knot $K \subset \Sigma$ has irreducible exterior $M=M(K)$ and if $F$ is an essential surface in $M$ with $\partial F \neq \emptyset$, then all the components of $\partial F$ are mutually parallel non-trivial simple closed curves in $\partial M$. Thus all the components of $\partial F$ determine the same pair of primitive elements of $H_{1}(\partial M ; \mathbb{Z})$, differing by sign; these will be called the boundary classes of $F$. If we fix a framing of $K$, all the components of $\partial F$ have the same slope $p / q \in \mathbb{Q} \cup\{\infty\}$, called the boundary slope of $F$. We define an element $p / q$ of $\mathbb{Q} \cup \infty$ to be a boundary slope of $K$ if it is the boundary slope of an some bounded essential surface in $M$. We call $p / q$ a strict boundary slope if it is the boundary slope of some bounded strict essential surface in $M$. Given a framing, we define $\mathcal{B} K \subset \mathbb{Q} \cup\{\infty\}$ to be the set of all boundary slopes of $K$, and 
$\mathcal{B}_{s} K$ to be the set of strict boundary slopes of $K$.

According to a theorem of Hatcher's [6], $\mathcal{B} K$ is a finite set; hence so is $B s K$. Changing the framing of $K$ has the effect of replacing $\mathcal{B} K$ (resp. $\mathcal{B}_{s} K$ ) by its image under an element of $D_{\infty}$. In particular, if $\infty \notin \mathcal{B} K$ (resp. $\left.\infty \notin \mathcal{B}_{s} K\right)$, the diameter of $\mathcal{B} K$ (resp. $\mathcal{B}_{s} K$ ), which is defined to be the difference between the greatest and least elements, is an invariant of the knot $K$.

If $U_{0} \subset \Sigma$ is a solid torus, we define a cable on $U_{0}$ to be a simple closed curve in $\partial U_{0}$ which has geometric intersection number at least 2 with a meridian of $\partial U_{0}$. A knot $K$ in $\Sigma$ is called a cable knot if it is not round and is a cable on some solid torus $U_{0}$. (If $U_{0}$ is a regular neighborhood of a knot $K_{0}$ we may also say that $K$ is obtained by cabling $K_{0}$.)

We may now state our main result.

Theorem 1.1. Let $\Sigma$ be an orientable 3-manifold with cyclic fundamental group, and let $K$ be a knot in $\Sigma$ such that $M(K)$ is irreducible and $\infty \notin \mathcal{B}_{s} K$. If $K$ is not a cable knot or a round knot then $\operatorname{diam} \mathcal{B}_{s} K \geq 2$.

1.2. Theorem 1.1 can be paraphrased by saying that if $K$ is not round and is not a cable knot, and $M(K)$ is irreducible and $\infty \notin \mathcal{B}_{s} K$, then in terms of any framing of $K$ there exist elements $s_{1}, s_{2} \in \mathcal{B}_{s} K$ such that $\left|s_{1}-s_{2}\right| \geq 2$. Of course the quantity $\left|s_{1}-s_{2}\right|$ is independent of the framing since it is invariant under the action of $D_{\infty}$ described above. However, it is instructive, and will be useful for the proof of Theorem 1.1, to give a direct definition of this quantity which does not involve the choice of a framing. To this end let us denote by $\langle\cdot, \cdot\rangle$ the intersection pairing on $H_{1}(\partial M ; \mathbb{Q})$. This pairing is determined by a choice of orientation of $\partial M$, and is in any case well-defined up to sign. Now if $F_{1}$ and $F_{2}$ are bounded essential surfaces in $M(K)$, then with each $F_{i}$ we can associate a boundary class $\alpha_{i}$, well-defined up to sign. Given any framing $(\mu, \nu)$ of $K$ we can write $\alpha_{i}$ in the form $p_{i} \mu+q_{i} \nu$ for $i=1,2$, and the boundary slope of $F_{i}$ is $s_{i}=p_{i} / q_{i}$. We then have

$$
\left|s_{1}-s_{2}\right|=\left|\frac{p_{1} q_{2}-p_{2} q_{1}}{q_{1} q_{2}}\right|=\left|\frac{\left\langle\alpha_{1}, \alpha_{2}\right\rangle}{\left\langle\alpha_{1}, \mu\right\rangle\left\langle\alpha_{2}, \mu\right\rangle}\right|,
$$

where the expression on the right is clearly independent of all choices of sign. If we now define a strict boundary class for $M(K)$ to be the boundary class of a strict essential surface then Theorem 1.1 can be reformulated as follows:

Theorem 1.3. Let $\Sigma$ be an orientable 3-manifold with cyclic fundamental group, and let $K$ be a knot in $\Sigma$. Suppose that $M=M(K)$ is irreducible and that $K$ is not a cable knot or a round knot. Let $\mu \in H_{1}(M ; \mathbb{Z})$ denote a meridian of $K$. Then either $\mu$ is a strict boundary class for $M$, or there exist strict boundary classes $\alpha_{1}$ 
and $\alpha_{2}$ for $M$ such that

$$
\left|\frac{\left\langle\alpha_{1}, \alpha_{2}\right\rangle}{\left\langle\alpha_{1}, \mu\right\rangle\left\langle\alpha_{2}, \mu\right\rangle}\right| \geq 2 .
$$

Example 1.4. The following example, which was discovered by Nathan Dunfield, shows that Theorem 1.1 is sharp.

Let $T$ be a compact orientable genus-1 surface with one boundary component. We identify the mapping class group of $T$ with $P S L(2, \mathbb{Z})$. Let $M$ denote the bundle over $S^{1}$ with fiber $T$ and monodromy

$$
\left(\begin{array}{cc}
0 & 1 \\
-1 & -3
\end{array}\right)
$$

(This manifold is sometimes called the sister of the figure- 8 complement.)

The fundamental group of $M$ has a presentation

$$
\left|a, b, t: t^{-1} a t=a b a^{-1}, t^{-1} b t=b^{-3} a^{-1}\right| .
$$

The commuting elements $t$ and $a b a^{-1} b^{-1}$ of $\pi_{1}(M)$ are represented by peripheral simple closed curves whose homology classes, respectively denoted $\tau$ and $\lambda$, form a basis for $H_{1}(\partial M ; \mathbb{Z})$. We will work in terms of the framing $(\tau, \lambda)$. We may use the algorithm in [2] to find the essential surfaces in $M$. In the notation of [2], we find three surfaces: the fiber, $C(1: 3)$ and $C(-1:-3)$. The "standard framing" determined by $C(1: 3)$ is the framing $(\tau, \lambda)$, so according to $[2$, Table I], the surface $C(1: 3)$ has boundary class $\alpha_{1}=4 \tau+\lambda$. The "transition index" between the standard framing of $C(-1:-3)$ and that of $C(1: 3)$ is 1 , so the boundary slope of $C(-1:-3)$ turns out to be $\alpha_{2}=4 \tau+3 \lambda$.

Next we claim that the manifold $\Sigma$ obtained by Dehn filling of $M$ along a curve representing the class $\mu=2 \tau+\lambda$ has cyclic fundamental group. We have

$$
\pi_{1}(\Sigma)=\left|a, b, t: t^{-1} a t=a b a^{-1}, t^{-1} b t=b^{-3} a^{-1}, a b a^{-1} b^{-1} t^{2}=1\right| .
$$

(Note that $t$ and $t^{-1}$ were interchanged in the description of the standard framing that appears in [2].) Substituting $t^{-1} a t$ for $a b a^{-1}$ in the third relation we obtain

$$
\pi_{1}(\Sigma)=\left|a, b, t: t^{-1} a t=a b a^{-1}, t^{-1} b t=b^{-3} a^{-1}, a t b^{-1} t=1\right| .
$$

Since the third relator implies $a=t^{-1} b t^{-1}$ we may eliminate the generator $a$. This gives

$$
\begin{aligned}
\pi_{1}(\Sigma) & =\left|b, t: t^{-1} b=b t^{-1} b t b^{-1} t, t^{-1} b=b^{-3} t b^{-1}\right| \\
& =\left|b, t: t^{-1} b t^{-1} b t^{-1} b^{-1} t b^{-1}=1, t^{-1} b^{2} t^{-1} b^{2}=b^{-1}\right|
\end{aligned}
$$

Writing $u=t^{-1} b^{2}$ and eliminating $t$ we have

$$
\pi_{1}(\Sigma)=\left|u, b: u b^{-1} u b^{-1} u b^{-1} u^{-1} b^{-1}=1, u^{2}=b^{-1}\right|
$$


Finally we may eliminate $b$ to obtain

$$
\pi_{1}(\Sigma)=\left|u: u^{10}=1\right| .
$$

Thus $M$ is the exterior of a knot $K$ in the manifold $\Sigma$. The fundamental group of $\Sigma$ is cyclic and the meridian of $K$ is $\mu$. We have

$$
\left|\frac{\left\langle\alpha_{1}, \alpha_{2}\right\rangle}{\left\langle\alpha_{1}, \mu\right\rangle\left\langle\alpha_{2}, \mu\right\rangle}\right|=\left|\frac{8}{2(-2)}\right|=2
$$

and, for $i=1,2$,

$$
\left|\frac{\left\langle\alpha_{i}, \lambda\right\rangle}{\left\langle\alpha_{i}, \mu\right\rangle\langle\lambda, \mu\rangle}\right|=1 .
$$

Thus $\mathcal{B} K$ has diameter 2 .

We remark that the manifolds obtained by $2 / 1$ and $1 / 0$ Dehn filling also have cyclic fundamental groups (of order 5 instead of 10). Thus the manifold $M$ is also extremal with respect to the Cyclic Surgery Theorem [1].

The following result, which will be proved at the end of Section 3, gives a lower bound for the diameter of $\mathcal{B} K$ in the case of most cable knots $K$, provided that $H_{1}(\Sigma ; \mathbb{Z})$ is finite.

If $U_{0}$ is a solid torus in a closed orientable 3 -manifold $\Sigma$, and if the meridian and external longitude of $U_{0}$ have geometric intersection number at least 2 , then the external longitude can be viewed as a cable on $U_{0}$. Such a cable will termed exceptional. A knot in $\Sigma$ will be called an exceptional cable knot if it is not round and is an exceptional cable on some solid torus $U_{0} \subset \Sigma$. Notice that in a homology sphere there are no exceptional cable knots.

Proposition 1.5. Let $\Sigma$ be a closed orientable 3-manifold such that $H_{1}(\Sigma ; \mathbb{Z})$ is finite, and let $K$ be a cable on a solid torus $U_{0} \subset \Sigma$. Suppose that $M(K)$ is irreducible (or, equivalently, that $\overline{\Sigma-U_{0}}$ is irreducible), and that $\infty \notin \mathcal{B K}$. Let $n$ be the order of the homology class in $H_{1}(\Sigma ; \mathbb{Z})$ represented by a core curve of $U_{0}$. Then either $K$ is a round knot, or $K$ is an exceptional cable on $U_{0}$, or $\operatorname{diam} \mathcal{B} K \geq 2 / n$.

Combining Proposition 1.5 with Theorem 1.1 we obtain the following result.

Corollary 1.6. Let $K$ be a knot in a closed 3-manifold $\Sigma$ with finite cyclic fundamental group. Suppose that $M(K)$ is irreducible and that $\infty \notin \mathcal{B} K$. Then either $K$ is a round knot or an exceptional cable knot, or $\operatorname{diam} \mathcal{B} K \geq 2 /\left|\pi_{1}(\Sigma)\right|$.

As we observed above, there are no exceptional cable knots in a homology 3sphere. Moreover, if a knot $K$ in a homotopy 3 -sphere $\Sigma$ is round, then $\Sigma$ is $S^{3}$ 
and $K$ is a trivial knot. Thus Corollary 1.6 specializes to the following result in the case that $\Sigma$ is a homotopy 3 -sphere.

Corollary 1.7. Let $K$ be a knot in a homotopy 3 -sphere $\Sigma$, with $M(K)$ irreducible and $\infty \notin \mathcal{B} K$. Then either $\operatorname{diam} \mathcal{B} K \geq 2$ or $\Sigma$ is $S^{3}$ and $K$ is a trivial knot.

1.8. Corollary 1.7 does not extend to knots in an arbitrary homology 3 -sphere. To see this, let us consider any non-trivial oriented $\operatorname{knot} K$ in $S^{3}$, and let $\Sigma_{n}$ be the result of $1 / n$ Dehn surgery along $K$. By definition this means that $\Sigma_{n}$ is obtained from $M=M(K)$ by attaching a solid torus $U_{n}$ along the boundary in such a way that the boundary of an essential disk in $U_{n}$ is identified with a simple closed curve whose slope, in terms of the standard framing $(\mu, \lambda)$ of $K$, is $1 / n$. A core curve of $U_{n}$ is then a knot $K_{n} \subset \Sigma_{n}$. Furthermore, $\Sigma_{n}$ is a homology 3-sphere, and $K_{n}$ can be oriented so that its standard framing is $\left(\mu_{n}, \lambda\right)$, where $\mu_{n}=\mu+n \lambda$. If $F$ is an essential surface in $M$ whose boundary slope, in terms of the standard framing of $K$, is $p / q$, then the boundary curves of $F$ can be oriented so as to represent the homology class $p \mu+q \lambda=p \mu_{n}+(q-p n) \lambda$; thus the boundary slope of $F$, in terms of the standard framing of $K_{n}$, is $p /(q-p n)$. As this quantity approaches 0 when $n$ tends to infinity, it follows from Hatcher's finiteness theorem that $\operatorname{diam}\left(\mathcal{B} K_{n}\right)$ also approaches 0 as $n \rightarrow \infty$.

1.9. In order to explain the potential relevance of Theorem 1.1 to the Poincaré Conjecture, it is convenient to restate it in a contrapositive form. Let $\Sigma$ be a closed orientable 3 -manifold. Suppose that $\Sigma$ contains a knot $K$ with the following property:

(*) $\quad M(K)$ is irreducible, $K$ is not cabled, $\infty \notin \mathcal{B} K$, and diam $\mathcal{B} K<2$.

In this situation, Theorem 1.1 asserts that if $\pi_{1}(\Sigma)$ is cyclic, then $K$ must be a round knot. According to our definition, this implies that $M$ is a solid torus, so that $\Sigma$ is homeomorphic to a (possibly trivial) lens space or to $S^{2} \times S^{1}$.

We express this by saying that $(*)$ is a "good property" of a knot. In general, let $\mathrm{Z}$ be a property of knots in closed, orientable 3-manifolds. We will say that $\mathrm{Z}$ is good if every knot $K$ with property $\mathrm{Z}$ in a closed, orientable 3-manifold with cyclic fundamental group is round.

While $(*)$ is a good property, it follows from 1.8 that many irreducible nonHaken manifolds contain knots with Property $(*)$. If one can exhibit a good property Z such that every non-Haken manifold contains a knot with Property Z, the Poincaré Conjecture will follow; more generally, it will follow that every closed 3 -manifold $\Sigma$ with finite cyclic fundamental group is a lens space. (This deduction is immediate in the case where $\Sigma$ is irreducible, since a manifold with finite cyclic fundamental group is non-Haken. The general case follows from Kneser's finiteness theorem.) 


\section{Norms}

In the case where $K$ is a hyperbolic knot, Theorem 1.1 follows via elementary arguments from the properties of the norm on $H_{1}(\partial M(K) ; \mathbb{R})$ that were established in [1] and used in the proof of the Cyclic Surgery Theorem. In the case where $M(K)$ contains an incompressible torus we need to decompose $M(K)$ into hyperbolic and Seifert-fibered pieces and use a slightly more general version of this norm to analyze the hyperbolic pieces. The results in [1] apply to manifolds with a single torus boundary component, but here we need to consider manifolds with several torus boundary components. The $S L_{2}(\mathbb{C})$-character varieties of such manifolds were considered in [4], but the norm was not introduced in that context.

In this section we sketch the construction of this generalized norm and enumerate its fundamental properties. We shall use the notation of [1], modified slightly to accommodate a manifold which may have more than one cusp.

Throughout this section we shall assume that $N$ is an orientable 3-manifold which is homeomorphic to the compact core of a finite-volume hyperbolic 3manifold. We shall also assume that $\partial N$ consists of tori $B_{0}, \ldots, B_{k}$, where $k \geq 0$. The manifold $N$ plays the rôle of the manifold $M$ which is discussed in [1], with the torus component $B_{0}$ of $\partial N$ replacing the torus $\partial M$. Accordingly, we identify the group $L=H_{1}\left(B_{0} ; \mathbb{Z}\right)$ with a lattice in the vector space $V=H_{1}\left(B_{0} ; \mathbb{R}\right)$. We let $e: L \rightarrow \pi_{1}\left(B_{0}\right)$ denote the inverse of the Hurewicz map; we often view $e$ as a homomorphism from $L$ to $\pi_{1}(M)$ which is well defined up to composition with inner automorphisms. If $\alpha$ is a primitive element of $L$ then we denote by $N(\alpha)$ the manifold obtained by glueing a solid torus along $B_{0}$ so that the meridian of the solid torus is identified with a curve representing $\alpha$.

We consider the complex affine algebraic set $R=R\left(\pi_{1}(N)\right)$ of representations of $\pi_{1}(N)$ in $S L_{2}(\mathbb{C})$. The set of characters of such representations is also an affine algebraic set and the map $\rho \mapsto \chi_{\rho}$ is a polynomial map. To each element $\gamma$ of $\pi_{1}(N)$ there is associated a function $I_{\gamma}$ on $X(N)$ defined by $I_{\gamma}(\chi)=\chi(\gamma)$ for every character $\chi \in X(N)$.

For each $i=1, \ldots, k$ we choose a peripheral element $\gamma_{i} \neq 1$ in the image of $\pi_{1}\left(B_{i}\right)$ in $\pi_{1}(N)$. We may consider the affine algebraic subset $W=W\left(\gamma_{1}, \ldots, \gamma_{n}\right)$ of $X(N)$ defined by the equations $I_{\gamma_{i}}= \pm 2$, for $i=1, \ldots, n$. It is shown in [4, page 541, proof of Theorem 3] that there exists a curve $C_{0}$ contained in $W$ such that

(C1) $C_{0}$ contains the character $\chi_{0}$ of a discrete faithful representation of $\pi_{1}(N)$.

It is also shown that for any such curve $C_{0}$ we have

(C2) for $i=1, \ldots, n$, if $\gamma \in \pi_{1}(N)$ is a peripheral element conjugate to an element of $\pi_{1}\left(B_{i}\right)$ then $I_{\gamma}$ is identically equal to either 2 or -2 on $C_{0}$; and

(C3) if $\gamma \neq 1$ is conjugate to an element of $\pi_{1}\left(B_{0}\right)$ then $I_{\gamma}$ is non-constant. 
2.1. We let $\widetilde{C}_{0}$ denote the smooth projective model of the curve $C_{0}$. This curve will replace the curve $\widetilde{X}_{0}$ in [1]. As in [1] there is associated to each ideal point $x$ of $\widetilde{C}_{0}$ a non-trivial action of $\pi_{1}(N)$ on the tree for $S L_{2}(F)$, where $F$ is the function field of $\widetilde{C}_{0}$ equipped with the discrete valuation $v_{x}$ associated with the smooth point $x$. In this section we shall denote this tree by $T_{x}$. We shall be considering surfaces in $N$ which are associated to the action of $\pi_{1}(N)$ on $T_{x}$ in the sense of [1, Definition 1.3.1]. The statements of Propositions 1.2.6, 1.2.7 and 1.3.2 of [1] remain true with $N$ replacing $M$ and $\widetilde{C}_{0}$ replacing $\widetilde{X}_{0}$, and the proofs remain the same. In particular, no point of $T_{x}$ is fixed by $\pi_{1}(N)$ and therefore any surface associated with the action of $\pi_{1}(N)$ on $T_{x}$ is non-empty.

The main modification that we must make to the results of [1] consists of replacing the class of essential surfaces in $M$ with the class of essential surfaces in $N$ which are disjoint from the boundary tori $B_{1}, \ldots, B_{k}$. This is made possible by the following

Lemma 2.2. If $x$ is any ideal point of $\widetilde{C}_{0}$ then for each $i=1, \ldots, k$ the image of $\pi_{1}\left(B_{i}\right)$ in $\pi_{1}(N)$ is contained in the stabilizer of some vertex of $T_{x}$

(As in [1] we shall often work with groups that are defined only up to conjugacy when there is no danger of confusion.)

Proof. For any $\gamma \in \pi_{1}\left(B_{i}\right)$, for $i \geq 1$, the function $I_{\gamma}$ is constant on on $\widetilde{C}_{0}$. Thus by [1, Proposition 1.2.6] the element $\gamma$ fixes a vertex of $T$. It is shown in [13, Corollary 3 to Proposition 26] that a finitely generated group fixes a vertex of a tree if each of its elements fixes a vertex.

The next lemma is a slight generalization of Proposition 1.3.8 of [1]. The proof is identical.

Lemma 2.3. Assume that $\pi_{1}(N)$ acts on a tree $T$ and that no vertex of $T$ is fixed by the entire group. Then there exists an essential surface associated to the action. Furthermore, if $K$ is a subcomplex of $\partial N$ such that for each component $K^{\prime}$ of $K$ the image of $\pi_{1}\left(K^{\prime}\right)$ in $\pi_{1}(N)$ is contained in a vertex stabilizer, then the surface may be taken to be disjoint from $K$.

Generalizing the definition from [1] we define a strict boundary class to be an element of $L$ which is the boundary class of an essential surface $F$ such that $\partial F \subset B_{0}$ and such that $F$ is not isotopic to the fiber of a fibration of $N$ over $S^{1}$. (The last condition is automatic if $k \geq 1$.)

The next lemma generalizes Proposition 1.3.9 of [1]

Lemma 2.4. Let $x$ be an ideal point of $\widetilde{C}_{0}$. Let $\alpha$ be a primitive element in $L$ such that $I_{e(\alpha)}(x) \neq \infty$. Then either $I_{e(\beta)}(x) \neq \infty$ for all $\beta \in L$ or else $\alpha$ is a 
strict boundary class.

Proof. By [1, Proposition 1.2.6] $e(\alpha)$ stabilizes a vertex of $T_{x}$. By Lemma 2.2, for $i=1, \ldots, k$ the image of $\pi_{1}\left(B_{i}\right)$ in $\pi_{1}(N)$ also fixes a vertex of $T_{x}$. We apply Lemma 2.3 taking $K=B_{1} \cup \cdots \cup B_{k} \cup c$, where $c$ is a simple closed curve $B_{0}$ representing $\alpha$. This gives an essential surface $S$ associated to the action of $\pi_{1}(N)$ on $T_{x}$ such that $\partial S$ is contained in $B_{0}$ and is disjoint from $c$. We now proceed exactly as in the proof of Proposition 1.3.9 of [1].

If $f$ is an element of the function field $\mathbb{C}\left(C_{0}\right)=\mathbb{C}\left(\widetilde{C}_{0}\right)$ we will denote by $Z_{x}(f)$ the order of zero of $f$ at the point $x \in \widetilde{C}_{0}$. If $f(x) \neq 0$ we set $Z_{x}(f)=0$. Similarly we denote by $\Pi_{x}(f)$ the order of pole of $f$ at $x$, with $\Pi_{x}(f)=0$ if $f$ does not have a pole at $x$. Thus $v_{x}(f)=Z_{x}(f)-\Pi_{x}(f)$. For each element $\alpha$ of $L$ we will let $f_{\alpha}$ denote the function $f_{\alpha}=I_{e(\alpha)}^{2}-4$, which vanishes wherever $I_{e(\alpha)}$ takes the value 2 or -2 .

The next lemma generalizes Proposition 1.6.1 of [1].

Lemma 2.5. Let $x$ be an ideal point of $\widetilde{C}_{0}$. Let $\alpha$ and $\delta$ be non-zero elements of L. Suppose that $\alpha$ is primitive and is not a strict boundary class, and that

$$
Z_{x}\left(f_{\alpha}\right)>Z_{x}\left(f_{\delta}\right)
$$

Then $M$ contains a closed orientable $\pi_{1}$-injective surface of positive genus. In particular $\pi_{1}(M(\alpha))$ is not cyclic.

Note that the conclusion that $\pi_{1}(\alpha)$ is not cyclic is non-trivial only in the case where $N$ has at most two boundary components. The case of two boundary components will be crucial for our application of this lemma.

Proof of Lemma 2.5. It suffices to produce a closed surface associated to the action of $\pi_{1}(N)$ on $T_{x}$. Then the proof of [1, Proposition 1.6.1], which occupies Section 1.6 of [1], goes through without change.

By applying Lemma 2.3 with $K=\partial N$ we see that there will exist a closed surface if the image of $\pi_{1}\left(B_{i}\right)$ fixes a vertex of $T_{x}$ for $i=0, \ldots, k$. We know from Lemma 2.2 that the image of $\pi_{1}\left(B_{i}\right)$ fixes a vertex of $T_{x}$ for $i \geq 1$. Our hypothesis implies that $I_{e(\alpha)}(x) \neq \infty$. Since $\alpha$ is not a strict boundary class, we conclude from Lemma 2.4 that $I_{e(\beta)}(x) \neq \infty$ for all $\beta \in L$. Arguing as in the proof of Lemma 2.2 this implies that the image of $\pi_{1}\left(B_{0}\right)$ fixes a vertex of $T_{x}$.

We may view $\widetilde{C}_{0}$ and $\mathbb{P}^{1}$ as compact 2-manifolds and regard $f_{\alpha}$ as a branched covering map from $\widetilde{C}_{0}$ to $\mathbb{P}^{1}$. As such, $f_{\alpha}$ has a well-defined topological degree which equals the number of inverse images of a generic point of $\mathbb{P}^{1}$. The following result generalizes Proposition 1.1.2 and Corollary 1.1.4 of [1]. 
Proposition 2.6. There exists a norm $\|\cdot\|$ on the vector space $V$ which has the following properties.

(N1) For every $\alpha \in L,\|\alpha\|$ is equal to the degree of $f_{\alpha}$.

(N2) The unit ball of the norm $\|\cdot\|$ is a (balanced convex) polygon. Each vertex of this polygon is a rational multiple of a strict boundary class (in $L \subset V)$.

(N3) If $\alpha$ is a primitive class in $L$ which is not a strict boundary class and if, in addition, the Dehn-filled manifold $N(\alpha)$ has cyclic fundamental group, then $\|\alpha\| \leq\|\delta\|$ for each non-zero element $\delta$ of $L$.

Proof. The proof is essentially the same as the proofs of the corresponding statements in [1], using Lemmas 2.4 and 2.5 in place of Propositions 1.3.9 and 1.6.1 of [1]. We sketch the main ideas.

Let $\alpha$ be an element of $L$. We first observe that the degree of $f_{\alpha}$ can be computed as the sum, over all ideal points $p$ of $\widetilde{C}_{0}$, of the order of pole of $f_{\alpha}$ at $p$. (The function $f_{\alpha}$ has no poles at ordinary points of $\widetilde{C}_{0}$.) Thus we have

$$
\operatorname{deg} f_{\alpha}=\sum_{x \text { ideal }} \Pi_{x}\left(f_{\alpha}\right) .
$$

One shows, using the valuation extension theorem exactly as in [1, section 1.4], that for each ideal point $x$ of $\widetilde{C}_{0}$ there exists a homomorphism of abelian groups $\phi_{x}: L \rightarrow \mathbb{Z}$ such that $\Pi_{x}\left(f_{\alpha}\right)=\left|\phi_{x}(\alpha)\right|$ for every $\alpha$ in $L$. We extend $\phi_{x}$ to a linear functional $\Phi_{x}: V \rightarrow \mathbb{R}$ and for each $v \in V$ we set

$$
\|v\|=\sum_{x \text { ideal }}\left|\Phi_{x}(v)\right|
$$

In particular if $\alpha$ is an element of $L$ then $\|\alpha\|$ equals the degree of $f_{\alpha}$. Thus the equality in (N1) holds.

The function $\|\cdot\|$ is a sum of the absolute values of finitely many linear functionals with integer coefficients, each arising from an ideal point of $\widetilde{C}_{0}$. This implies that $\|\cdot\|$ is a linear semi-norm given by a piecewise-linear function with integer coefficients. If $\|\cdot\|$ were not a norm then there would exist a homomorphism non-zero element $\alpha$ of $L$ which was contained in the kernel of every $\phi_{x}$. But then the degree of $f_{\alpha}$ would be zero, i.e. $f_{\alpha}$ would be constant. This is ruled out by condition (C3) above, so $\|\cdot\|$ is a norm.

Each vertex $u$ of the unit ball of $\|\cdot\|$ is an element of the kernel of one of the linear functionals $\Phi_{x}$, where $\phi_{x}$ is not identically zero. Hence $u$ is a scalar multiple of a generator $\alpha$ of the kernel of $\phi_{x}$. Since $\phi_{x}(\alpha)=0$ we have that $f_{\alpha}(x) \neq \infty$. Since $\phi_{x}$ is not identically zero there exists $\beta$ in $L$ with $f_{\beta}(x)=\infty$. Thus by Lemma $2.4 \alpha$ is a strict boundary class. This establishes property (N2).

To prove property (N3) we use the observation that the degree of $f_{\alpha}$ can also be written as the sum of $Z_{x}\left(f_{\alpha}\right)$ over all points $x \in \widetilde{C}_{0}$. We will assume that 
$f_{\alpha}(x)=0$ and consider two cases, according to whether $x$ is an ideal point or not. In each case we will obtain a contradiction to the hypothesis that $\pi_{1}(N(\alpha))$ is cyclic.

Suppose that we are given two arbitrary elements $\alpha$ and $\delta$ of $L$. If $x$ is not an ideal point then we can apply Proposition 1.5.2 of [1], with $M$ replaced by $N$ and $X_{0}^{\nu}$ replaced by the set $\widetilde{C}_{0}^{\nu}$ of ordinary points of $\widetilde{C}_{0}$. The proof of this proposition, which occupies Section 1.5 of [1], goes through without change. The essential property of $C_{0}$ which is used in the argument is that it contains the character of an irreducible representation. We conclude that if $Z_{x}\left(f_{\alpha}\right)>Z_{x}\left(f_{\delta}\right)$ then there exists a representation of $\pi_{1}(N(\alpha))$ into $P S L_{2}(\mathbb{C})$ which has non-cyclic image. This is a contradiction. If $x$ is an ideal point then we can apply Lemma 2.5 to conclude that if $Z_{x}\left(f_{\alpha}\right)>Z_{x}\left(f_{\delta}\right)$ then $\pi_{1}(N(\alpha))$ is non-cyclic, giving a contradiction.

2.7. As in [1] it will be useful to consider the ball $Q=\{v:\|v\| \leq m\}$ where $m=\min _{0 \neq \delta \in L}\|\delta\|$. It follows from (N2) that this is a balanced convex polygon. By definition there are no non-zero elements of $L$ in its interior. By a theorem of Minkowski's $Q$ has area at most 4 with respect to the natural area element in which the area of $V / L$ is 1 ( $\operatorname{cf}[1$, page 244]). It follows from (N3) that if $\mu$ is a primitive element of $L$ such that $\pi_{1}(N(\mu))$ is cyclic then $\mu$ lies on the boundary of $Q$. If, in addition, $\mu$ is not a strict boundary class then (N2) implies that $\mu$ is not a vertex of $Q$ and hence lies in the interior of a side.

\section{Proof of the Main Theorem}

This section is devoted to the proof of Theorem 1.3 which, as was pointed out in Section 1, is equivalent to Theorem 1.1. Throughout the section $\Sigma$ will denote an orientable 3-manifold with cyclic fundamental group, $K$ will denote a knot in $\Sigma$ with irreducible complement, and $M=M(K)$ will denote the exterior of $K$. We shall assume that $K$ is not round. The solid torus $\Sigma-M$ will be denoted by $U$. We shall let $\mu$ denote a meridian of $K$.

According to the Characteristic Submanifold Theory of Jaco and Shalen [9] and Johannson [10], and Thurston's Geometrization Theorem [11], there exists, up to isotopy, an essential surface $\mathcal{T}_{M} \subset M$, each component of which is a torus, with the property that each component of the manifold $\mathcal{N}$ obtained by splitting $M$ along $\mathcal{T}_{M}$ is either a Seifert-fibered space or the compact core of a finite-volume hyperbolic manifold. We will think of $\mathcal{N}$ as being the complement of an open regular neighborhood of $\mathcal{T}_{M}$. Let $N_{P} \subset M$ denote the component of $\mathcal{N}$ that contains the boundary torus of $M$. We regard the boundary torus of $M$ as a distinguished component of $\partial N_{P}$ which we denote $B_{0}$. The components of the frontier $\partial N_{P}-B_{0}$ of $N_{P}$ are all tori. 
Lemma 3.1. Suppose that $\infty \notin \mathcal{B}_{s} K$. Then either $N_{P}=M$ or $N_{P} \cup U$ is a solid torus. In particular, $\pi_{1}\left(N_{P} \cup U\right)$ is cyclic and the frontier of $N_{P}$ has at most one connected component.

Proof. We shall assume that $N_{P} \neq M$ and show that $N_{P} \cup U$ is a solid torus. We can write $\Sigma$ as the union of the two submanifolds $N_{P} \cup U$ and $\overline{M-N_{P}}$ which meet along the frontier of $N_{P}$. Since $\mathcal{T}_{M}$ is essential each component of $\overline{M-N_{P}}$ is boundary irreducible. Since $\Sigma$ has cyclic fundamental group and therefore cannot contain a $\pi_{1}$-injective torus, we conclude that $N_{P} \cup U$ is boundary reducible. Thus $N_{P} \cup U$ has a solid torus connected summand. It therefore suffices to show that $N_{P} \cup U$ is irreducible.

Since $N_{P}$ is irreducible any 2-sphere which does not bound a ball in $N_{P} \cup U$ must meet $U$. Every isotopy class of 2-spheres in $N_{P} \cup U$ contains a sphere which meets the solid torus $U$ in meridian disks. Among all spheres which do not bound balls and which meet $U$ in meridian disks, choose one, say $S$, for which the number of meridian disks is minimal. Then $S \cap N_{P}$ is a properly embedded planar surface in $N_{P}$. We claim that $S \cap N_{P}$ is essential. If the inclusion $\pi_{1}\left(S \cap N_{P}\right) \rightarrow \pi_{1}\left(N_{P}\right)$ fails to be injective then we can use a compressing disk for $S \cap N_{P}$ to produce two 2-spheres in $N_{P} \cup U$. One of these spheres fails to bound a ball in $N_{P} \cup U$ and both meet $U$ in fewer meridian disks than $S$. We know that $S \cap N_{P}$ is not boundary parallel in $N_{P}$ because otherwise $S$ would bound a ball in $N_{P} \cup U$. The surface $S \cap N_{P}$ cannot be a fiber in a fibration of $M$ over $S^{1}$ because there is an essential torus in its complement. This shows that $\infty \in \mathcal{B}_{s} K$, in contradiction to our hypotheses.

Recall that the hypotheses of Theorem 1.3 specify that the knot $K$ is not a cable. The next lemma allows us to conclude under this hypothesis that $N_{P}$ is hyperbolic.

Lemma 3.2. Suppose that $\infty \notin \mathcal{B}_{s} K$. If $N_{P}$ is Seifert-fibered then $K$ is a cable knot.

Proof. We fix a Seifert fibration of $N_{P}$. We may assume that this is not a Seifert fibration over a Möbius band with no singular fibers since the twisted $I$-bundle over a Klein bottle also admits a Seifert fibration over a disk with two singular fibers. Because $\mathcal{T}_{M}$ consists of essential tori we also do not have a Seifert fibration over a disk with at most one singular fiber, nor a Seifert fibration over an annulus with no singular fibers.

Next we claim that the meridian is not a Seifert fiber. Let $X$ denote the base of our Seifert fibration and let $\beta_{0}$ be the boundary component of $X$ which is the image of the torus $B_{0}$ under the Seifert fibration. We may choose an $\operatorname{arc} \alpha$ in $X$, with $\partial \alpha \subset \beta_{0}$, which is essential in the sense that any closed disk having $\alpha$ as its frontier contains the image of at least one singular fiber. The inverse image of $\alpha$ 
under the Seifert fibration is an essential annulus in $N_{P}$ and hence in $M$. Our choice of Seifert fibration guarantees that this annulus is not a fiber in a fibration over $S^{1}$. Thus the Seifert fiber is a strict boundary slope and hence cannot be the meridian.

We may therefore extend the Seifert fibration of $N_{P}$ to a Seifert fibration of $N_{P} \cup U$ in such a way that $K$ is a fiber. By Lemma 3.1 we know that $N_{P} \cup U$ has a cyclic fundamental group. Thus the Seifert fibration must either have base $S^{2}$ with at most two singular fibers, or base $\mathbb{P}^{2}$ or $D^{2}$ with at most one singular fiber. In each of these cases our conditions on the Seifert fibration of $N_{P}$ guarantee that $K$ must be a regular fiber and that there must be at least one singular fiber in $N_{P}$. Let $D$ be a disk in the base of the Seifert fibration of $N_{P} \cup U$ containing the image of $K$ and the image of exactly one singular fiber. Then the inverse image of $D$ in $N_{P} \cup U$ is a solid torus $W$. Since $K$ is a regular fiber, it is isotopic to a curve on the boundary of $W$. If $n>1$ denotes the index of the singular fiber in $W$ then $K$ has geometric intersection number $n$ with a meridian disk of $W$, and hence $K$ is a cable.

For the rest of the section we assume that $K$ is not a cable knot, so that by Lemma 3.2 $N_{P}$ is homeomorphic to the compact core of a finite-volume hyperbolic 3-manifold. We shall follow the conventions of Section 2, taking $N=N_{P}$ and letting $B_{0}=\partial M$ be the distinguished boundary component of $N$. We set $V=$ $H_{1}\left(B_{0} ; \mathbb{R}\right)$ and $L=H_{1}\left(B_{0} ; \mathbb{Z}\right) \subset H_{1}\left(B_{0} ; \mathbb{R}\right)$. We shall work with the norm $\|\cdot\|$ on $V=H_{1}\left(B_{0} ; \mathbb{R}\right)$ which was constructed in Section 2 and, in particular, with the polygon $Q$ which was defined in 2.7. Recall that $Q=\{v:\|v\| \leq m\}$ where $m=\min _{0 \neq \delta \in L}\|\delta\|$.

In Section 2 we were considering essential surfaces in $N$ whose boundary is contained in $B_{0}$. The boundary classes of such surfaces are elements of $L$, as are boundary classes of essential surfaces in $M$. Recall from the definition that if $N_{P}$ has at least one frontier component, i.e. is not equal to $M$, and if $F$ is any essential surface in $N_{P}$ with $\partial F \subset B_{0}$, then the boundary class of $F$ is automatically a strict boundary class.

Lemma 3.3. An element of $L$ is a strict boundary class for $M$ if it is a strict boundary class for $N_{P}$.

Proof. We may assume that $N_{P}$ has non-empty frontier since otherwise $N_{P}=M$. Under this assumption we shall show that if $F$ is an essential surface in $N_{P}$ with $\partial F \subset B_{0}$ then $F$ is a strict essential surface in $M$. Because the frontier tori of $N_{P}$ are essential in $M$, and $F$ is essential in $N_{P}$, it follows that $F$ is essential in $M$. Since the complement of $F$ in $M$ contains esential tori, namely the frontier tori of $N_{P}$, the surface $F$ cannot be a fiber in a fibration of $M$ over $S^{1}$. Thus $F$ is a strict essential surface in $M$. 
Now we are ready to give the

Proof of Theorem 1.3. Assume that $\infty \notin \mathcal{B}_{s} K$. Then by Lemma 3.1 the manifold $N_{P} \cup U=N_{P}(\mu)$ has cyclic fundamental group. (Lemma 3.1 also implies that the number of frontier components of $N=N_{P}$, which was denoted by $k$ in Section 2, is at most 1.)

Recall from 2.7 that $Q$ is a balanced convex polygon of area at most 4 with respect to the canonical area element on $V$ such that $V / L$ has area 1 . Since $N_{P}(\mu)$ has cyclic fundamental group, we know from 2.7 that $\mu$ lies on the boundary of $Q$. Moreover, since $\mu$ is not a strict boundary class by Lemma 3.3 , we know from 2.7 that $\mu$ lies in the interior of a side of $Q$. Let $v_{1}$ and $v_{2}$ be the vertices of $Q$ that are the endpoints of the side containing $\mu$. By (N2) these are multiples of strict boundary classes $\alpha_{1}$ and $\alpha_{2}$. We shall show that

$$
\left|\frac{\left\langle\alpha_{1}, \alpha_{2}\right\rangle}{\left\langle\alpha_{1}, \mu\right\rangle\left\langle\alpha_{2}, \mu\right\rangle}\right| \geq 2
$$

We may extend the intersection pairing $\langle\cdot, \cdot\rangle$ on $L$ to an alternating bilinear pairing on the vector space $V$, also denoted $\langle\cdot, \cdot\rangle$. We then have

$$
\left|\frac{\left\langle\alpha_{1}, \alpha_{2}\right\rangle}{\left\langle\alpha_{1}, \mu\right\rangle\left\langle\alpha_{2}, \mu\right\rangle}\right|=\left|\frac{\left\langle v_{1}, v_{2}\right\rangle}{\left\langle v_{1}, \mu\right\rangle\left\langle v_{2}, \mu\right\rangle}\right| .
$$

We write $\mu=t v_{1}+(1-t) v_{2}$. We have

$$
\left\langle v_{1}, \mu\right\rangle=(1-t)\left\langle v_{1}, v_{2}\right\rangle \quad \text { and } \quad\left\langle v_{2}, \mu\right\rangle=-t\left\langle v_{1}, v_{2}\right\rangle .
$$

Since the parallelogram with vertices at $\pm v_{1}$ and $\pm v_{2}$ is contained in $Q$, its area, $2\left|\left\langle v_{1}, v_{2}\right\rangle\right|$, is at most 4 .

Hence

$$
\left|\frac{\left\langle v_{1}, v_{2}\right\rangle}{\left\langle v_{1}, \mu\right\rangle\left\langle v_{2}, \mu\right\rangle}\right|=\left|\frac{1}{t(t-1)\left\langle v_{1}, v_{2}\right\rangle}\right| \geq \frac{1}{2 t(1-t)} .
$$

The right hand side of this inequality is bounded below by 2 since the maximum value on $[0,1]$ of the function $t(1-t)$ is $1 / 4$. This completes the proof.

We end by giving the

Proof of Proposition 1.5. Let $M_{0}=\overline{\Sigma-U_{0}}$ and let $\left(\mu_{0}, \nu_{0}\right)$ be a framing of $U_{0}$. Let $p \mu_{0}+q \nu_{0} \in H_{1}\left(\partial U_{0} ; \mathbb{Z}\right)$ be the class represented by the knot $K$.

We may choose the regular neighborhood $U(K)$ of $K$ so that the boundary torus of $U_{0}$ meets $M=M(K)=\overline{\Sigma-U(K)}$ in an annulus $A$. The boundary curves of $A$ are parallel in $\partial U_{0}$ to the knot $K$, and thus have slope $p / q$ on $\partial U_{0}$. We claim that either $K$ is a round knot or $A$ is an essential annulus in $M_{0}$. If $A$ is compressible, then the knot $K$ bounds a disk in $\Sigma$. But, since $\Sigma$ is irreducible, 
this implies that $\Sigma$ is a 3 -sphere and $K$ a trivial knot. In particular, $K$ is round. On the other hand, if $A$ is boundary-parallel then, since $K$ is a cable on $U_{0}$ and hence meets the meridian of $U_{0}$ at least twice, $A$ must be parallel to the annulus $\partial M \cap M_{0}$. This implies that $M_{0}$ is a solid torus whose core is parallel to $K$. Again, this shows that $K$ is round. This proves the claim. We may therefore assume that $A$ is essential.

Let $\alpha$ be a class in $H_{1}(\partial U(K) ; \mathbb{Z})$ represented by the boundary components of $A$, and let $\mu$ be a meridian of $U(K)$. Note that $(\mu, \alpha)$ is a framing of $K$. Since we have assumed that $A$ is essential, $\alpha$ is a boundary class for $M$.

Let $\bar{\mu}, \bar{\mu}_{0}$ and $\bar{\nu}_{0}$ and $\bar{\alpha}$ denote the elements of $H_{1}(M ; \mathbb{Z})$ which are the images under inclusion of $\mu, \mu_{0}$ and $\nu_{0}$ and $\alpha$ respectively. In particular, we have $\bar{\alpha}=$ $p \bar{\mu}_{0}+q \bar{\nu}_{0}$. Moreover, we have the relation $q \bar{\mu}=\bar{\mu}_{0}$. To see this, consider a solid torus $U^{\prime}$ which is isotopic to $U(K)$ by a small isotopy and is contained in the interior of $U_{0}$. Let $D$ be a meridian disk of $U_{0}$ which meets $U^{\prime}$ in $q$ meridian disks of $U^{\prime}$. Since the meridian of $U^{\prime}$ represents the class $\mu$, the planar surface $\overline{D-U^{\prime}}$ exhibits the relation in question.

Since $H_{1}(\Sigma ; \mathbb{Z})$ is finite, the image of $\nu_{0}$ under the inclusion homomorphism $H_{1}\left(M_{0} ; \mathbb{Z}\right) \rightarrow H_{1}(\Sigma ; \mathbb{Z})$ is an element of some finite order $n$. Note that $n$ is equal to the order of a class in $H_{1}(\Sigma ; \mathbb{Z})$ which is represented by a core curve of $U_{0}$. Since $H_{1}(\Sigma ; \mathbb{Z})$ is the quotient of $H_{1}\left(M_{0} ; \mathbb{Z}\right)$ by the cyclic subgroup generated by the image of $\mu_{0}$, we have $m \bar{\mu}_{0}+n \bar{\nu}_{0}=0$ for some integer $m$. In particular the external longitude of $U_{0}$ has slope $m / n$ with respect to the framing $\left(\mu_{0}, \nu_{0}\right)$. Since $K$ is a non-exceptional cable it follows that $m / n \neq p / q$, so $\Delta=p n-q m$ is non-zero.

Let us write the external longitude of $K$ as $a \mu+b \alpha$ where $a$ and $b$ are arbitrary integers. Then $a \bar{\mu}+b \bar{\alpha}$ is a torsion element in $H_{1}(M ; \mathbb{Z})$. On the other hand

$$
\begin{aligned}
n a \bar{\mu}+n b \bar{\alpha} & =n a \bar{\mu}+n b p \bar{\mu}_{0}+n b q \bar{\nu}_{0} \\
& =n a \bar{\mu}+n b p q \bar{\mu}-m b q^{2} \bar{\mu} \\
& =(n a+\Delta q b) \bar{\mu} .
\end{aligned}
$$

We know that the class $\bar{\mu}$ has infinite order, since otherwise $H_{1}(\Sigma)$ would be infinite. Thus we must have

$$
\frac{a}{b}=\frac{-\Delta q}{n}
$$

We have $|q| \geq 2$ by the definition of a cable. Thus $|a / b| \geq 2 / n$. With respect to the framing $(\mu, \alpha)$ of $K$ we have $0 \in \mathcal{B} K$ since $\alpha$ is the boundary class of an essential annulus, and $a / b \in \mathcal{B} K$ since $a \mu+b \nu$ is an external longitude of $K$. It follows that

$$
\operatorname{diam} \mathcal{B} K \geq|a / b| \geq 2 / n
$$




\section{References}

[1] M. Culler, C. McA. Gordon, J.Luecke and P. B. Shalen, Dehn surgery on knots, Ann. of Math. 125 (2) (1987), 237-300.

[2] M. Culler, W. Jaco and H. Rubinstein, Incompressible surfaces in once-punctured torus bundles, Proc. London Math. Soc. 45 (3) (1982), 385-419.

[3] M. Culler and P. B. Shalen, Varieties of Representations and splittings of 3-manifolds, Ann. of Math. 59 (1984), 197-207.

[4] M. Culler and P. B. Shalen, Bounded, separating incompressible surface in knot manifolds, Invent. Math. 84 (1984), 537-545.

[5] N. Dunfield, Cyclic surgery, degrees of maps of character curves, and volume rigidity for hyperbolic manifolds, preprint, University of Chicago.

[6] A. Hatcher, On the boundary slopes of incompresible surfaces, Pacific J. Math. 99 (1982), 373-377.

[7] A. Hatcher and U. Oertel, Boundary slopes for Montesinos knots, Topology 28 (1989), $453-480$.

[8] A. Hatcher and W. Thurston, Incompressible surfaces in 2-bridge knot complements, Invent. Math. 79 (1985), 225-246.

[9] W. H. Jaco and P. B. Shalen, Seifert-fibered spaces in 3-manifolds, Mem. Amer. Math. Soc. 21 (1979), no. 220

[10] K. Johannson, Homotopy equivalences of 3-manifolds with boundaries, Lecture Notes in Mathematics, no. 761 Springer, Berlin 1979.

[11] J.-P. Otal, Thurston's hyperbolization of Haken manifolds. Prépublication no. 208, Ecole Normale Supérieure de Lyon 1997.

[12] P. B. Shalen, Handbook of Geometric Topology (R. Daverman and R. Sher, eds.), NorthHolland Publishing Co., Amsterdam (to appear).

[13] J. P. Serre (with H. Bass), Trees, Springer-Verlag, New York 1980.

Marc Culler and Peter B. Shalen

Department of Mathematics, Statistics

and Computer Science (MC 249)

University of Illinois at Chicago

851 S. Morgan St.

Chicago, IL 60607-7045

USA

e-mail: culler@math.uic.edu

(Received: January 20, 1998) 